\title{
PSEUDO-ORBITS, PSEUDOLEAVES AND GEOMETRIC ENTROPY OF FOLIATIONS
}

\author{
Andrzej Biś And PaWee WalczaK
}

November 4, 1996

\begin{abstract}
We show that the entropy of a finitely generated pseudogroup (resp., of a foliation on a compact Riemannian manifold) can be calculated by suitable counting separated pseudo-orbits (resp., pseudoleaves).
\end{abstract}

0. Introduction. Since several years, pseudo-orbits play an important role in the theory of classical dynamical systems. In particular, it is shown ([Mi] and [BS]) that pseudo-orbits can be used to calculate the topological entropy of transformations. More recently, the similar result was obtained [Hur] for the inverse-image entropy introduced earlier by Remi Langevin and the second author [LW1].

On the other hand, geometric entropy $h(\mathcal{F})$ of a foliation $\mathcal{F}$ of a compact Riemannian manifold has been introduced $[\mathbf{G L W}]$ and shown to be a handful tool to study topology and dynamics of foliated manifolds ( [Hu1 - 3], [GLW], [LW2], $[\mathbf{E g}],[\mathbf{I T}]$, etc.). The entropy $h(\mathcal{F})$ can be calculated either by counting the number of points separated along the leaves or by counting the number of separated orbits of holonomy pseudogroups generated by nice coverings by charts distinguished by $\mathcal{F}$. Also, pseudoleaves of foliations have been defined by Takashi Inaba $[\mathbf{I n}]$ who has shown (among the other results) that expansive $\mathrm{C}^{1}$-foliations (in the sense of [IT]) of codimension-one which have the pseudoleaf tracing property are topologically stable.

In this article, we show that the geometric entropy $h(\mathcal{F})$ of any foliation $\mathcal{F}$ of a compact Riemannian manifold $M$ coincides with that calculated by suitable counting separated pseudoleaves (Theorem 2 in Section 4). To this end, we study the entropy of finitely generated pseudogroups of local transformations of compact metric spaces and we show that it can be calculated by counting (again, in a suitable way) separated pseudo-orbits (Theorem 1 in Section 2). In Section 5, we sketch an easy proof of Theorem 2 for foliated bundles. Finally, in Section 6 we provide an example of a group acting on $S^{1}$ for which the "usual" formula for the entropy in terms of pseudo-orbits does not work: It gives the number strictly bigger than the entropy calculated in terms of separated orbits.

Some significance of these results could be observed if one would try to calculate (or, to estimate) entropies of pseudogroups or foliations with the aid of computers. 
The authors are grateful to Takashi Inaba for his valuable comments made during his visit to the University of Eódź.

1. Pseudo-orbits of pseudogroups. Let $(X, d)$ be a compact metric space and $G$ be a finitely generated pseudogroup of local transformations of $X$. For any $g \in G$, $U_{g} \subset X$ will denote the domain of $g$. Let us fix a finite symmetric generating set $G_{1} \subset G$. Recall that "symmetric" means that $e=\operatorname{id}_{X} \in G_{1}$ and $G_{1}^{-1} \subset G_{1}$. For any $k \in \mathbb{N}$ let $G_{k}=\left(G_{1}\right)^{k}=\left\{\left(h_{1}, \ldots h_{k}\right) ; h_{i} \in G_{1}\right\}$. We shall identify $G_{k}$ with a subset of $G_{k+1}$ consisting of all the sequences of the form $\left(e, h_{1}, \ldots h_{k}\right)$, $h_{i} \in G_{1}$. Also, given two sequences $h=\left(h_{1}, \ldots h_{k}\right) \in G_{k}$ and $g=\left(g_{1} \ldots g_{l}\right) \in G_{l}$ we shall denote by $(h, g)$ the element $\left(h_{1}, \ldots h_{k}, g_{1}, \ldots g_{l}\right) \in G_{k+l}$. Finally, for any $h=\left(h_{1}, \ldots k_{k}\right) \in G_{k}$ we shall denote by the same symbol $h$ the map $h_{1} \circ \cdots \circ h_{k} \in G$. Therefore, we may write that $\{e\}=G_{0} \subset G_{1} \subset G_{2} \subset \cdots \subset G_{\infty}=\cup_{k} G_{k}$.

The entropy of the system $\left(G, G_{1}\right)$ can be defined whenever the maps of $G$ are homeomorphisms between open sets. However, in general it can be infinite and the most natural setting ensuring its finiteness is the category of locally Lipschitz maps. Therefore, throughout the paper we shall assume that the maps generating pseudogroups are Lipschitz.

Let us fix $\alpha \geq 0$. An $\alpha$-pseudo-orbit of $G$ is a map $x: D_{x} \rightarrow X$ such that $D_{x} \subset G_{\infty}, e \in D_{x}$, for any $h \in G_{1}$ and $g \in D_{x},(h, g) \in D_{x}$ iff $x(g) \in U_{h}, U_{h}$ being the domain of $h$, and then

$$
d(h(x(g)), x(h, g)) \leq \alpha .
$$

If $G$ is a group of global transformations, then $D_{x}=G_{\infty}$ for any pseudo-orbit $x$ and inequality (1) should be satisfied for all $g \in G_{\infty}$ and $h \in G_{1}$. In particular, if $G=\mathbb{Z}$ acts on $X$ and $f$ is a generator, then $x: \mathbb{Z} \rightarrow X$ is just a sequence of points and (1) reduces to

$$
d\left(f\left(x_{n}\right), x_{n+1}\right) \leq \alpha, \quad n \in \mathbb{Z}
$$

i. e. $x$ becomes a pseudo-orbit of $f$ in the classical sense [Bo].

Let us denote by $Y_{\alpha}$ the space of all the $\alpha$-pseudo-orbits. Clearly, $Y_{\alpha} \subset Y_{\beta}$ whenever $\alpha \leq \beta$ and $Y_{0}=\cap_{\alpha \geq 0} Y_{\alpha}$ is the space of "real" $G$-orbits. Recall, that $x \in Y_{0}$ iff $x=x_{p}$ for some $p \in X, D_{x_{p}}=\left\{g \in G_{\infty} ; p \in U_{g}\right\}$ and $x_{p}(g)=g(p)$ for all $g \in D_{x_{p}}$.

For any pseudo-orbits $x$ and $y$ in $Y_{\alpha}$ put

$$
d_{0}(x, y)=\sum_{k=0}^{\infty} \frac{1}{2^{k}} \max \left\{d(x(g), y(g)) ; g \in G_{k} \cap D_{x} \cap D_{y}\right\}
$$

and

$$
d_{1}(x, y)=\inf \left\{\sum_{i=0}^{m-1} d_{0}\left(z_{i}, z_{i+1}\right) ; z_{i} \in Y_{\alpha}, z_{0}=x, z_{m}=y \text { and } m \in \mathbb{N}\right\}
$$

Lemma 1. For any $\alpha,\left(Y_{\alpha}, d_{1}\right)$ is a metric space.

Proof. The symmetry and triangle inequality for $d_{1}$ as well as the relation $d_{1}(x, x)=$ 0 for all $x \in Y_{\alpha}$ follow immediately from (2) and (3). It remains to show that 
the condition $d_{1}(x, y)=0$ implies the equality $x=y$ which is equivalent to the following:

$$
D_{x} \cap G_{k}=D_{y} \cap G_{k} \text { and } x=y \text { on } D_{x} \cap D_{y} \cap G_{k}
$$

for $k=0,1,2, \ldots$

First, observe that

$$
\sum_{i=0}^{m-1} d_{0}\left(z_{i}, z_{i+1}\right) \geq \sum_{i=0}^{m-1} d\left(z_{i}(e), z_{i+1}(e)\right) \geq d(x(e), y(e))
$$

whenever $z_{0}=x$ and $z_{m}=y$. Therefore, the condition $d_{1}(x, y)=0$ implies that $x(e)=y(e)$ and then (4) is satisfied with $k=0$.

Next, observe that the equality $x(e)=y(e)=p$ implies that $D_{x} \cap G_{1}=D_{y} \cap G_{1}=$ $G(p) \cap G_{1}$, where $G(p)=\left\{h \in G ; p \in U_{h}\right\}$. Find $\epsilon>0$ such that $B(p, \epsilon) \subset U_{h}$ for all $h \in G(p) \cap G_{1}$. If $z_{0}, z_{1}, \ldots, z_{m} \in Y_{\alpha}, z_{0}=x, z_{m}=y$ and $\sum_{i} d_{0}\left(z_{i}, z_{i+1}\right)<\frac{\epsilon}{2}$, then

$$
d\left(p, z_{j}(e)\right) \leq \sum_{i \leq j} d\left(z_{i}(e), z_{i+1}(e)\right) \leq \sum_{i \leq j} d_{0}\left(z_{i}, z_{i+1}\right)<\frac{\epsilon}{2}
$$

and $z_{j}(e) \in U_{h}$ for any $h \in G(p) \cap G_{1}$ and $j \leq m$. Therefore,

$$
d(x(h), y(h)) \leq \sum_{i} d\left(z_{i}(h), z_{i+1}(h)\right) \leq 2 \cdot \sum_{i} d_{0}\left(z_{i}, z_{i+1}\right)<\epsilon
$$

for all $h \in G(p) \cap G_{1}$. This shows that the condition $d_{1}(x, y)=0$ implies (4) with $k=1$.

Proceed by induction to complete the proof.

Lemma 2. The space $\left(Y_{\alpha}, d_{1}\right)$ is compact.

Proof. Take any sequence $\left(x_{n}\right)$ of $\alpha$-pseudo-orbits. Choose a subsequence $\left(x_{n}^{0}\right)$ of $\left(x_{n}\right)$ such that $x_{n}^{0}(e) \rightarrow p \in X$. Put $x(e)=p$ and $D_{x} \cap G_{1}=G(p) \cap G_{1}$. Find a subsequence $\left(x_{n}^{1}\right)$ of $\left(x_{n}^{0}\right)$ such that $x_{n}^{1}(h) \rightarrow p_{h} \in X$ for any $h \in D_{x} \cap G_{1}$. Put $x(h)=p_{h}$ and $D_{x} \cap G_{2}=\left\{\left(h^{\prime}, h\right) ; h \in D_{x} \cap G_{1}, h^{\prime} \in G_{1}\right.$ and $\left.p_{h} \in U_{h^{\prime}}\right\}$. Proceed by induction to define $D_{x} \cap G_{k}$, to find subsequences $\left(x_{n}^{k}\right)$ such that $x_{n}^{k}(g) \rightarrow p_{g} \in X$ for all $g \in D_{x} \cap G_{k}, k \in \mathbb{N}$, and put $x(g)=p_{g}$ for all such $g$.

If $h \in D_{x} \cap G_{1}$, then $h \in D_{x_{n}^{0}}$ and $d\left(x_{n}^{0}(h), h\left(x_{n}^{0}(e)\right) \leq \alpha\right.$ for all $n$ large enough. Therefore, $d(x(h), h(x(e))) \leq \alpha$. Also, if $\left(h^{\prime}, h\right) \in D_{x} \cap G_{2}$, then $h^{\prime} \in D_{x_{n}^{1}}$ and $d\left(x_{n}^{1}\left(h^{\prime}, h\right), h^{\prime}\left(x_{n}^{1}(h)\right)\right) \leq \alpha$ for all $n$ large enough. Therefore, $d\left(x\left(h^{\prime}, h\right), h^{\prime}(x(h))\right) \leq$ $\alpha$. Proceed by induction to show that $x \in Y_{\alpha}$.

Obviously, $x_{n}^{n} \rightarrow x$.

Now, take $g_{0} \in G_{\infty}$ and $x \in Y_{\alpha}$ such that $g_{0} \in D_{x}$. Define $y=\sigma_{g_{0}}(x) \in Y_{\alpha}$ by

$$
y(g)=x\left(g, g_{0}\right)
$$

on $D_{y}=\left\{g \in G_{\infty} ;\left(g, g_{0}\right) \in D_{x}\right\}$. It is easy to see that this definition is correct and that all the maps $\sigma_{h}, h \in G_{1}$, generate a pseudogroup, denoted by $G$ again, of local transformations of $Y_{\alpha}$. 
Note that

$$
\begin{aligned}
d_{0}\left(\sigma_{g_{0}} x, \sigma_{g_{0}} x^{\prime}\right) & =\sum_{k} \frac{1}{2^{k}} \max \left\{d\left(x\left(g, g_{0}\right), x^{\prime}\left(g, g_{0}\right)\right) ; g \in G_{k} \cap D_{x} \cap D_{x^{\prime}}\right\} \\
& \leq \sum_{k} \frac{1}{2^{k}} \max \left\{d\left(x(g), x^{\prime}(g)\right) ; g \in G_{k+n} \cap D_{x} \cap D_{x^{\prime}}\right\} \leq 2^{n} d_{0}\left(x, x^{\prime}\right)
\end{aligned}
$$

and

$$
\begin{aligned}
d_{1}\left(\sigma_{g_{0}} x, \sigma_{g_{0}} x^{\prime}\right) & \left.=\inf \left\{\sum_{i \leq m-1} d_{0}\left(z_{i}, z_{i+1}\right) ; z_{0}=\sigma_{g_{0}} x \text { and } z_{m}=\sigma_{g_{0}} x^{\prime}\right)\right\} \\
& \leq \inf \left\{\sum_{i \leq m-1} d_{0}\left(\sigma_{g_{0}} z_{i}, \sigma_{g_{0}} z_{i+1}\right) ; z_{0}=x \text { and } z_{m}=x^{\prime}\right\} \leq 2^{n} d_{1}\left(x, x^{\prime}\right)
\end{aligned}
$$

whenever $g_{0} \in G_{n}$. Therefore, $G$ acts on $Y_{\alpha}$ also via locally Lipschitz transformations.

2. Entropy of pseudogroups. As before, let $G$ be a pseudogroup on $X$ equipped with a finite symmetric generating set $G_{1}$. Following $[\mathbf{G L W}]$ we shall say that two points $p$ and $q$ of $X$ are $(n, \epsilon)$-separated w. r. t. $G(n \in \mathbb{N}, \epsilon>0)$ if there exists $g \in G_{n}$ such that $\{p, q\} \subset U_{g}$ and $d(g(p), g(q)) \geq \epsilon$. A set $A \subset X$ is $(n, \epsilon)$-separated when any two points $p, q \in A, p \neq q$, have this property. Since $X$ is compact, separated sets are always finite and we may put

$$
\begin{gathered}
N(n, \epsilon, X)=\max \{\# A ; A \subset X \text { is }(n, \epsilon) \text {-separated }\}, \\
N(\epsilon, X)=\limsup _{n \rightarrow \infty} \frac{1}{n} \log N(n, \epsilon, X)
\end{gathered}
$$

and

$$
h\left(G, G_{1}, X\right)=\lim _{\epsilon \rightarrow 0^{+}} N(\epsilon, X) .
$$

The number $h\left(G, G_{1}, X\right)$ is the entropy of $G$ (w. r. t. $\left.G_{1}\right)$ on $X$.

Lemma 3. $h\left(G, G_{1}, X\right)=h\left(G, G_{1}, Y_{0}\right)$, $Y_{0}$ being the space of $G$-orbits.

Proof. The inequality " $\leq$ "follows immediately from the estimate

$$
d_{1}\left(\sigma_{g} x, \sigma_{g} y\right) \geq d(x(g), y(g))=d(g(p), g(q))
$$

which holds for the orbits $x$ and $y$ of any points $p$ and $q$ of $X$ and any element $g \in G_{\infty}$ such that $\{p, q\} \subset U_{g}$.

To prove " $\geq$ " take two $(n, \epsilon)$-separated orbits $x$ and $y \in Y_{0}$ and find $g \in G_{n}$ such that $\{x(e), y(e)\} \subset U_{g}$ and $d_{1}\left(\sigma_{g}(x), \sigma_{g}(y)\right) \geq \epsilon$. Then also $d_{0}\left(\sigma_{g}(x), \sigma_{g}(y)\right) \geq \epsilon$. Next, find $l(\epsilon) \in \mathbb{N}$ such that $\sum_{k>l(\epsilon)} 2^{-k}<\epsilon /(2 \operatorname{diam} X)$. Thus,

$$
d\left(g^{\prime} g(x(e)), g^{\prime} g(y(e))\right)=d\left(x\left(g^{\prime}, g\right), y\left(g^{\prime}, g\right)\right)=d\left(\sigma_{g} x\left(g^{\prime}\right), \sigma_{g} y\left(g^{\prime}\right)\right) \geq \frac{\epsilon}{2}
$$

for some $g^{\prime} \in G_{l(\epsilon)}$ and the points $x(e), y(e)$ are $(n+l(\epsilon), \epsilon / 2)$-separated on $X$. Consequently,

$$
N\left(n, \epsilon, Y_{0}\right) \leq N\left(n+l(\epsilon), \frac{\epsilon}{2}, X\right) .
$$

Pass to suitable limits to complete the proof. 
Lemma 4. For any $n \in \mathbb{N}$ and any $\epsilon>0$ there exists $\alpha>0$ such that $N\left(n, \frac{\epsilon}{3}, Y_{0}\right) \geq$ $N\left(n, \epsilon, Y_{\alpha}\right)$.

Proof. Fix $n \in \mathbb{N}$ and $\epsilon>0$. Let $L$ be the Lipschitz constant for all the generators of $G$. Let $A=\left\{x_{i}, i \leq k\right\}$ be an $(n, \epsilon)$-separated subset of $Y_{\alpha}$. For any $i$, let $x_{i}^{\prime}$ be the orbit of $x_{i}(e)$. An easy induction shows that

$$
d\left(x_{i}(g), x_{i}^{\prime}(g)\right) \leq \alpha \cdot\left(1+L+\cdots+L^{n-1}\right)
$$

for any $i$ and any $g \in G_{n}$. Therefore, for any $i$ and $j \leq k$ there exists $g \in G_{n}$ such that

$$
\begin{aligned}
d\left(x_{i}^{\prime}(g), x_{j}^{\prime}(g)\right) & \geq d\left(x_{i}(g), x_{j}(g)\right)-d\left(x_{i}(g), x_{i}^{\prime}(g)\right)-d\left(x_{j}(g), x_{j}^{\prime}(g)\right) \\
& \geq \epsilon-2 \alpha \cdot\left(1+L+\cdots+L^{n-1}\right) \geq \frac{\epsilon}{3}
\end{aligned}
$$

whenever $\alpha$ is small enough to satisfy $\alpha \cdot\left(1+L+\cdots+L^{n-1}\right) \leq \frac{\epsilon}{3}$. For such $\alpha$, $A^{\prime}=\left\{x_{i}^{\prime}, i \leq k\right\} \subset Y_{0}$ is $(n, \epsilon / 3)$-separated.

Now, let us say that $\alpha$-pseudo-orbits $x$ and $y$ are $(n, \epsilon)$-strongly separated just when $d(x(g), y(g)) \geq \epsilon$ for some $g \in G_{n} \cap D_{x} \cap D_{y}$. Let us denote by $N_{\alpha}(n, \epsilon)$ the maximal cardinality of a set $A \subset Y_{\alpha}$ such that any two elements $x, y \in A, x \neq y$, are $(n, \epsilon)$-separated in this sense. Note that in general $N_{\alpha}(n, \epsilon) \neq N\left(n, \epsilon, Y_{\alpha}\right)$ since strong separation of $\alpha$-pseudo-orbits in the above sense is not entirely equivalent to separation of points of $Y_{\alpha}$ by the action of $G$. For any sequence $\beta=\left(\beta_{n}\right)$ such that $0<\beta_{n} \searrow 0$ we shall put

$$
\begin{gathered}
N_{\beta}(\epsilon)=\limsup _{n \rightarrow \infty} \frac{1}{n} \log N_{\beta_{n}}(n, \epsilon), \\
N_{\mathrm{ps}}(\epsilon)=\inf _{\beta} N_{\beta}(\epsilon)
\end{gathered}
$$

and

$$
h_{\mathrm{ps}}\left(G, G_{1}, X\right)=\lim _{\epsilon \rightarrow 0^{+}} N_{\mathrm{ps}}(\epsilon) .
$$

Our main result (Theorem 1) in this Section says that this "pseudo-entropy" $h_{\mathrm{ps}}$ coincides always with the "real " entropy $h$.

Lemma 5. $N_{\alpha}(n, \epsilon) \leq N\left(n, \epsilon, Y_{\alpha}\right)$ for all $n \in \mathbb{N}, \epsilon>0$ and $\alpha \geq 0$.

Proof. If pseudo-orbits $x, y \in Y_{\alpha}$ are $(n, \epsilon)$-strongly separated and $d(x(g), y(g)) \geq \epsilon$ for some $g \in G_{n} \cap D_{x} \cap D_{y}$, then

$$
d_{1}\left(\sigma_{g} x, \sigma_{g} y\right) \geq d\left(\sigma_{g} x(e), \sigma_{g} y(e)\right)=d(x(g), y(g)) \geq \epsilon,
$$

i. e. $x$ and $y$ are separated under the action of $G$ on $Y_{\alpha}$. 
Theorem 1. For any pseudogroup $G$ on $X$ and any finite symmetric generating set $G_{1}$ the equality

$$
h_{\mathrm{ps}}\left(G, G_{1}, X\right)=h\left(G, G_{1}, X\right)
$$

holds.

Proof. The inequality " $\geq$ " in (11) is obvious since $Y_{0} \subset Y_{\alpha}$ for all $\alpha \geq 0$ and the notions of separation of points of $X$ under the action of $G$ and strong separation of orbits just coincide.

To prove " $\leq$ " observe that, by Lemmas 4 and 5 ,

$$
\begin{aligned}
N_{\mathrm{ps}}(\epsilon) \leq N_{\beta}(\epsilon) & \leq \limsup _{n \rightarrow \infty} \frac{1}{n} \log N\left(n, \epsilon, Y_{\beta_{n}}\right) \\
& \leq \limsup _{n \rightarrow \infty} \frac{1}{n} \log N\left(n, \frac{\epsilon}{3}, Y_{0}\right)=N\left(\frac{\epsilon}{3}, Y_{0}\right)
\end{aligned}
$$

for any sequence $\beta=\left(\beta_{n}\right)$ such that $\beta_{n} \leq \epsilon \cdot\left(1+L+\cdots+L^{n-1}\right)^{-1}, n \in \mathbb{N}$ and $L$ being - as before - the Lipschitz constant for all the generators of $G$. Therefore,

$$
h_{\mathrm{ps}}\left(G, G_{1}, X\right) \leq h\left(G, G_{1}, Y_{0}\right)=h\left(G, G_{1}, X\right)
$$

by Lemma 3 .

Remark. The proof above shows that

$$
h\left(G, G_{1}, X\right)=\lim _{\epsilon \rightarrow 0^{+}} \lim _{n \rightarrow \infty} \frac{1}{n} \log N_{\epsilon \alpha_{n}}(n, \epsilon)
$$

with $\alpha_{n}=\left(1+L+\cdots+L^{n}\right)^{-1}$.

3. Pseudoleaves and holonomy pseudo-orbits. First, assume that $E$ is a vector space with an inner product $\langle\cdot, \cdot\rangle$. For any $q \geq 1$ and codimension- $q$ subspaces $E_{1}$ and $E_{2}$ of $E$ put

$$
\operatorname{dist}\left(E_{1}, E_{2}\right)=\max \left\{\min \left\{\left\|v_{1}-v_{2}\right\| ; v_{2} \in E_{2},\left\|v_{2}\right\|=1\right\} ; v_{1} \in E_{1},\left\|v_{1}\right\|=1\right\} .
$$

Clearly, dist is a distance function on the set of all codimension- $q$ subspaces of $E$ which is nearly proportional to the angle between subspaces.

Let $\mathcal{F}$ be a $\mathrm{C}^{r}$-foliation, $r \geq 1$, of a compact Riemannian manifold $(M,\langle\cdot, \cdot\rangle)$. Following [In] we shall say that a complete submanifold $N$ of $M$ is an $\alpha$-pseudoleaf when $\operatorname{dim} N=\operatorname{dim} \mathcal{F}$ and

$$
\operatorname{dist}\left(T_{p} N, T_{p} \mathcal{F}\right) \leq \alpha
$$

for all $p \in N$.

Next, take a nice cover $\mathcal{U}$ of $(M, \mathcal{F})[\mathbf{H H}]$ and denote by $H_{\mathcal{U}}$ the holonomy pseudogroup of $\mathcal{F}$ acting on the space $X=X_{\mathcal{U}}$ of plaques of the closed charts $\bar{U}$, $U \in \mathcal{U}$. Since $\mathcal{U}$ is finite, $X$ is compact.

The space $X$ can be equipped with some natural distance functions $d=d_{\mathcal{U}}$ induced from the Riemannian structure of $M$. For example, one can equip $M$ with the Riemannian distance function $d_{M}$ and define $d_{\mathcal{U}}$ as the Hausdorff distance 
between the closures of the plaques. Or, one can fix a complete transversal $T=T_{\mathcal{U}}$ intersecting each plaque exactly once, identify $X$ with $T$ and equip $T$ with the Riemannian distance function coming from the induced Riemannian structure. All these choices are equivalent in the sense that any two metrics described above are quasi-isometric and therefore the entropies of $H_{\mathcal{U}}$ obtained from these metrics are equal ([GLW], Prop. 2.6). So, let us fix such a "good" metric $d$ on $X$ once for ever and use it hereafter all the time.

Also, we consider the "standard" generating set $\left(H_{\mathcal{U}}\right)_{1}$ for $H_{\mathcal{U}}$. It consists of all the elementary holonomy maps $h_{U, V}$ corresponding to overlapping charts $U, V \in \mathcal{U}$ and defined by the following:

$$
h_{U, V}(P)=Q \text { iff the plaques } P \subset U \text { and } Q \subset V \text { overlap. }
$$

Since this is the only generating set to be considered, we shall omit the symbol $\left(H_{\mathcal{U}}\right)_{1}$ in all the formulae below.

Now, observe that the space of pseudoleaves embeds naturally into the space of pseudo-orbits of some holonomy pseudogroups $H_{\mathcal{U}}$. To produce such an embedding fix a complete transversal $T_{\mathcal{U}}$ and a subbundle $E$ of $T M$ complementary to $T \mathcal{F}$ and such that $E_{p}=T_{p} T_{\mathcal{U}}$ for any $p \in T_{\mathcal{U}}$. Also, for any two overlapping charts $U, V \in \mathcal{U}$ fix another distinguished chart $W=W_{U, V}$ containing $\bar{U} \cup \bar{V}$ and assume that $\mathcal{U}$ is tiny enough for all the plaques of the charts $W_{U, V}$ to be strongly convex subsets of the leaves.

Lemma 6. There exist positive constants $C, C^{\prime}$ and $\alpha_{0}$ such that for any $\alpha \leq \alpha_{0}$, any $\alpha$-pseudoleaf $N$ and any $p \in N$ there exists an unique $(C \alpha)$-pseudo-orbit $x$ of $H_{\mathcal{U}}$ such that $x(e)=p, x\left(D_{x}\right) \subset N$ and $d_{N}(x(h, g), x(g)) \leq C^{\prime} d_{\mathcal{F}}(h(x(g)), x(g))$ for any $g \in D_{x}$ and any generator $h$ of $H_{\mathcal{U}}$ such that $x(g) \in U_{h}$.

Proof. Define $x$ on $D_{x} \cap G_{k}$ inductively. First, $x$ is already defined on $D_{x} \cap G_{0}=\{e\}$ : $x(e)=p$. Then, if $g \in D_{x} \cap G_{k}, x(g)$ is already defined, $h=h_{U, V}$ is a generator of $H_{\mathcal{U}}$ and $x(g)$ lies in the domain of $h$, then join $x(g)$ to $h(x(g))$ by the unique leaf geodesic $\gamma:[0,1] \rightarrow W=W_{U, V}$, built from the fibres of the bundle $E$ a tubular neighbourhood $E_{\gamma}$ of a leaf $L_{p}$ around $\gamma([0,1])$ and lift $\gamma$ to the unique curve $\tilde{\gamma}:[0,1] \rightarrow E_{\gamma}$ such that $\pi_{\gamma} \circ \tilde{\gamma}=\gamma, \tilde{\gamma}(0)=p$ and $\tilde{\gamma}(t) \in N$ for all $t \in[0,1], \pi_{\gamma}$ being the canonical projection in the tubular neighbourhood. Finally, put $x(h, g)=\tilde{\gamma}(1)$ (see Figure 1 with $g=e$ ).

Since the lengths of all such geodesics $\gamma$ are uniformly bounded, this construction can be performed for $\alpha$ small enough, say $\alpha \leq \alpha_{0}$. Also, the same reason together with uniform bounds for the geometry (i. e., sectional curvature, injectivity radius etc.) of $M$ implies that $d_{M}(x(h, g), h x(g)) \leq C \alpha$ and that $l(\tilde{\gamma}) \leq C^{\prime} l(\gamma)$ for some universal constants $C$ and $C^{\prime}$. Therefore, this construction leads to an $C \alpha$-pseudoorbit of $H_{\mathcal{U}}$ which satisfies all the required conditions.

The uniqueness of such an $x$ follows from the fact that at each step of induction the construction is in fact unique if $\alpha$ is as small that the projections $\pi_{\gamma} \mid N$ are oneto-one for all minimal geodesics joining $q$ to $h(q)$ for any $q \in M$ and any holonomy map $h$ generating $H_{\mathcal{U}}$.

Remark. Clearly, any orbit of the holonomy pseudogroup $\mathcal{H}_{\mathcal{U}}$ is contained in a leaf of $\mathcal{F}$. It seems that embedding a holonomy $\alpha$-pseudo-orbit $x$ into a pseudoleaf is not so obvious. The difficulty comes from the fact that, even if $\alpha$ is very small, the points $x\left(h_{1}\right)$ and $x\left(h_{2}\right), h_{1}, h_{2} \in\left(\mathcal{H}_{\mathcal{U}}\right)_{1}$, may belong to a disc orthogonal to $\mathcal{F}$ (see 
Figure 2, where $q=h_{1}(p)=h_{2}(p)$ and $\left.p_{i}=x\left(h_{i}\right)\right)$ and therefore any submanifold $N$ containing these points in a reasonable way has to make an angle close to $\frac{\pi}{2}$ with some leaves of $\mathcal{F}$. However, in the case of foliated bundles, one can produce [In] immersed pseudoleaves corresponding to pseudo-orbits of the global holonomy group by suitable use of centres of mass $[\mathbf{B K}]$ of the points of pseudo-orbits.

4. Geometric entropy. Let again $(M, \mathcal{F},\langle\cdot, \cdot\rangle)$ be a compact foliated Riemannian manifold, $\mathcal{U}$ - a nice cover of $(M, \mathcal{F})$ and $T=T_{\mathcal{U}}$ - a complete transversal. Recall that two points $p, q \in T$ are $(R, \epsilon)$-separated w. r. t. $\mathcal{F}$ if either $d_{M}(p, q) \geq \epsilon$ or there exists a leaf curve $\gamma:[0,1] \rightarrow M$ of length $l(\gamma) \leq R$ and such that $\gamma(0)=p$ (resp., $\gamma(0)=q$ ), the originated at an $\mathcal{U}$-plaque through $q$ (resp., through $p$ ) orthogonal projection $\tilde{\gamma}$ of $\gamma$ onto the leaf through $q$ (resp., through $p$ ) exists and satisfies the condition

$$
d_{M}(\gamma(1), \tilde{\gamma}(1)) \geq \epsilon,
$$

$d_{M}$ being, as before, the Riemannian distance function on $M$. As usually, a subset $A$ of $T$ is $(R, \epsilon)$-separated when any two its points have this property. Since $M$ is compact, separated sets are always finite and we may define $N(R, \epsilon, \mathcal{F})$ as the maximal cardinality of an $(R, \epsilon)$-separated (w. r. t. $\mathcal{F})$ set. The geometric entropy $h(\mathcal{F})$ of $\mathcal{F}$ is defined by (see $[\mathbf{G L W}]$ and $[\mathbf{L W} \mathbf{2}]$ )

$$
h(\mathcal{F})=\lim _{\epsilon \rightarrow 0} N(\epsilon, \mathcal{F})
$$

where

$$
N(\epsilon, \mathcal{F})=\limsup _{R \rightarrow \infty} \frac{1}{R} \log N(R, \epsilon, \mathcal{F}) .
$$

Obviously, $h(\mathcal{F})$ depends on the Riemannian metric $\langle\cdot, \cdot\rangle$ on $M$ or, more precisely, on the Riemannian metric $\langle\cdot, \cdot\rangle \mid T \mathcal{F} \otimes T \mathcal{F}$ along the leaves: If two Riemannian metrics coincide on $T \mathcal{F}$, then the corresponding entropies are equal.

It is well known that the geometric entropy $h(\mathcal{F})$ is strongly related to the entropies of the holonomy pseudogroups:

$$
h(\mathcal{F})=\sup _{\mathcal{U}} \frac{1}{\Delta(\mathcal{U})} h\left(H_{\mathcal{U}}\right)
$$

where $\mathcal{U}$ ranges over the family of all nice covers of $(M, \mathcal{F})$ and $\Delta(\mathcal{U})$ is the smallest upper bound for the diameters of the plaques of $\mathcal{U}([\mathbf{G L W}]$, Thm. 3.4).

Note that, since any point $p$ determines the leaf $L_{p}$ uniquely, we can consider $N(R, \epsilon, \mathcal{F})$ as the number of separated leaves $L$ with base points $p \in T \cap L$. This approach can be adapted to the case of pseudoleaves even if there are infinitely many pseudoleaves passing through a given point.

Fix $\alpha_{0}>0$. Again since $M$ is compact and has bounded geometry, there exist positive numbers $\epsilon_{0}$ and $\epsilon_{1}$ such that for any points $p_{1}, p_{2} \in M$ for which $d_{M}\left(p_{1}, p_{2}\right)<\epsilon_{0}$ and any $\alpha$-pseudoleaves $N_{1}$ and $N_{2}$ with $\alpha \leq \alpha_{0}$ and $p_{i} \in N_{i}$ there exists an unique vector $v \in T_{p_{1}}^{\perp} N_{1}$ such that $p_{1}^{\prime}=\exp _{M}(v) \in N_{2}$ and $d_{N_{2}}\left(p_{1}^{\prime}, p_{2}\right)<\epsilon_{1}$. The point $p_{1}^{\prime}$ can be considered as the orthogonal projection of $p_{1}$ onto $N_{2}$. 
So, let us say that $\alpha$-pseudoleaves $\left(N_{i}, p_{i}\right)\left(\alpha \leq \alpha_{0}, i=1,2\right)$ with base points $p_{i} \in N_{i} \cap T$ are $(R, \epsilon)$-separated if either $d_{M}\left(p_{1}, p_{2}\right) \geq \epsilon_{0}$ or there exists a curve $\gamma:[0,1] \rightarrow N_{1}$ (resp.,,$N_{2}$ ) originated at $p_{1}$ (resp., at $p_{2}$ ) of the length $l(\gamma) \leq R$ and such that its orthogonal projection $\tilde{\gamma}$ onto $N_{2}$ (resp., onto $N_{1}$ ) originated in the ball $B_{N_{2}}\left(p_{2}, \epsilon_{1}\right)$ (resp., in $B_{N_{1}}\left(p_{1}, \epsilon_{1}\right)$ ) exists and satisfies inequality (15). Analogously to $(16)$ and (17), let us put

$$
h_{\mathrm{ps}}(\mathcal{F})=\lim _{\epsilon \rightarrow 0^{+}} \inf _{\alpha} N_{\alpha}(\epsilon, \mathcal{F})
$$

where

$$
N_{\alpha}(\epsilon, \mathcal{F})=\limsup _{R \rightarrow \infty} \frac{1}{R} \log N_{\alpha(R)}(R, \epsilon, \mathcal{F})
$$

$\alpha: \mathbb{R}_{+} \rightarrow \mathbb{R}_{+}$is any function satisfying $\alpha(R) \rightarrow 0$ while $R \rightarrow \infty$, and $N_{\alpha(R)}(R, \epsilon, \mathcal{F})$ is the maximal number of pairwise $(R, \epsilon)$-separated $\alpha(R)$-pseudoleaves equipped with base points.

Since any leaf becomes an $\alpha$-pseudoleaf for any $\alpha \geq 0$, it is obvious that our "geometric pseudo-entropy" $h_{\mathrm{ps}}(\mathcal{F})$ is not less than the "real" geometric entropy $h(\mathcal{F})$. Below, we shall prove that, similarly as in the case of a pseudogroup, these two entropies are equal.

Theorem 2. For any compact foliated Riemannian manifold $(M, \mathcal{F},\langle\cdot, \cdot\rangle)$ the equality

$$
h_{\mathrm{ps}}(\mathcal{F})=h(\mathcal{F})
$$

holds.

Proof. As was observed, we have only to prove the inequality " $\leq$ " in (20).

To this end cover $M$ by "coins" as in the proof of Theorem 3.4 of $[$ GLW]. "Coins" are distinguished charts $U_{p}, p \in M$, built along the transversals $T_{p}=$ $\exp _{M} B^{\perp}\left(0_{p}, \rho\right), B^{\perp}\left(0_{p}, \rho\right)$ being the centred at the origin ball of radius $\rho$ in $T_{p}^{\perp} \mathcal{F}$, the orthogonal complement of $T_{p} \mathcal{F}$ in $T_{p} M$. More precisely,

$$
U_{p}=\cup_{q \in T_{p}} B^{\mathcal{F}}(q, \Delta),
$$

where $B^{\mathcal{F}}(q, \Delta)$ is a centred at $q$ ball of radius $\Delta$ in $L_{q}$, the leaf of $\mathcal{F}$ at $q$. Here, $\rho$ and $\Delta$ are sufficiently small such that the corresponding exponential maps are diffeomorphic on all the balls of radii $2 \rho$ and $2 \Delta$.

Choose a finite subset $Q$ of $M, Q=\left\{q_{1}, \ldots, q_{N}\right\}$, such that the corresponding charts $U_{q_{i}}, i=1, \ldots, N$, form a nice covering $\mathcal{U}_{\Delta}$. Obviously, $\Delta\left(\mathcal{U}_{\Delta}\right)=2 \Delta$. Also, fix a small number $\eta>0$ and choose $Q$ dense in $M$ so that any point of $M$ is at most $\eta / 2$ apart in the leaf distance from a point of $T=T_{\mathcal{U}_{\Delta}}$ and $\eta / 2$ apart in $d_{M}$-distance from one of the points $q_{j} \in Q$. The existence of such a set $Q$ is shown in full detail in $[\mathbf{G L W}]$.

Take $\alpha>0$ small enough, $\epsilon \in\left(0, \epsilon_{0}\right),(R, \epsilon)$-separated $\alpha$-pseudoleaves $\left(N_{i}, p_{i}\right)$, $i=1,2$, and the corresponding $C \alpha$-pseudo-orbits $x_{i}$ of $H_{\mathcal{U}_{\Delta}}$ (Lemma 6).

If $d_{M}\left(p_{1}, p_{2}\right) \geq \epsilon_{0}$, then $d\left(x_{1}(e), x_{2}(e)\right) \geq \epsilon_{0} \geq \epsilon$ and the pseudo-orbits $x_{1}$ and $x_{2}$ are $(n, \epsilon)$-separated for all $n \geq 1$. Otherwise, there exists a curve $\gamma:[0,1] \rightarrow N_{1}$, 
$\gamma(0)=p_{1}, l(\gamma) \leq R$, separating $N_{1}$ from $N_{2}$, i.e. such that $d(\gamma(1), \tilde{\gamma}(1)) \geq \epsilon$, where $\tilde{\gamma}$ is the suitable projection of $\gamma$ onto $N_{2}$. Shortening $\gamma$ if necessary, we may assume that $d(\gamma(1), \tilde{\gamma}(1))=\epsilon$ and $d(\gamma(t), \tilde{\gamma}(t)) \leq \epsilon$ for all $t$.

Split $\gamma$ into $n=[1+R /(2 \Delta-2 \eta)]$ pieces $\gamma_{j}$ of length $l\left(\gamma_{j}\right) \leq 2 \Delta-2 \eta$. Set $p_{0}^{\prime}=p_{1}$ and $p_{n+1}^{\prime}=\gamma(1)$, for each $j=1, \ldots, n$ denote by $p_{j}^{\prime}$ the midpoint of $\gamma_{j}$ and find indices $i(j) \in\{1, \ldots, N\}$ such that $d_{M}\left(p_{j}^{\prime}, q_{i(j)}\right) \leq \eta / 2$ for $j=0,1, \ldots, n+1$. Thus, if $\alpha$ is small enough, each piece $\gamma_{j}$ is contained in a chart $U_{j}=U_{q_{i(j)}} \in \mathcal{U}_{\Delta}$. Let $g=\left(h_{n}, \ldots, h_{0}\right)$, where $h_{j}=h_{U_{j} U_{j+1}}$ for $j=0,1, \ldots, n$.

From the construction of the pseudo-orbits $x_{i}$ it follows that $d_{N_{1}}\left(p_{j}^{\prime}, x_{1}\left(g_{j}\right)\right) \leq \eta$ when $g_{j}=\left(h_{j}, \ldots, h_{0}\right), j=0,1, \ldots, n$ and $\alpha$ is, again, small enough (see Figure 3 , where $r_{j}=x_{1}\left(g_{j}\right), r_{j+1}=x_{1}\left(g_{j+1}\right), s_{j}=x_{2}\left(g_{j}\right)$ and $\left.s_{j+1}=x_{2}\left(g_{j+1}\right)\right)$. It follows that $x_{1}\left(g_{j}\right) \in U_{j}$ for all $j$ if only $\eta$ is small with respect to $\Delta$ and $\rho$. In particular, $g \in D_{x_{1}}$. Also, $g \in D_{x_{2}}$ if only $\epsilon$ is small enough. Moreover, since $d_{N_{1}}\left(x_{1}(g), \gamma(1)\right) \leq \eta$ and the similar inequality holds for $\tilde{\gamma}(1)$ and $x_{2}(g)$ on $N_{2}$, then $d_{M}\left(x_{1}(g), x_{2}(g)\right) \geq C^{\prime \prime} \epsilon$, where $C^{\prime \prime}>0$ is an universal constant depending only on $\rho, \Delta, \eta$ and the geometry of $M$. Hence, the pseudo-orbits $x_{1}$ and $x_{2}$ of $H_{\mathcal{U}_{\Delta}}$ are $\left(n+1, C^{\prime \prime} \epsilon\right)$-strongly separated and consequently

$$
N_{\alpha}(R, \epsilon, \mathcal{F}) \leq N_{C \alpha}\left([2+R /(2 \Delta-2 \eta)], C^{\prime \prime} \epsilon, H_{\mathcal{U}_{\Delta}}\right)
$$

for $\alpha$ and $\epsilon$ small enough.

Now, pass to suitable limits to arrive at

$$
h_{\mathrm{ps}}(\mathcal{F}) \leq \frac{1}{2 \Delta-2 \eta} h_{\mathrm{ps}}\left(H_{\mathcal{U}_{\Delta}}\right) .
$$

Finally, apply Theorem 1 and relation (18) to obtain the inequality

$$
h_{\mathrm{ps}}(\mathcal{F}) \leq \frac{\Delta}{\Delta-\eta} h(\mathcal{F})
$$

which yields the required result since $\eta>0$ may be arbitrarily small.

5. Groups and foliated bundles. If $G$ is a group of global transformations of $X$, then one need not take care of domains of maps, $D_{x}=G_{\infty}$ for any pseudo-orbit $x, d_{0}=d_{1}$ is defined simply by

$$
d_{0}(x, y)=d_{1}(x, y)=\sum_{k=0}^{\infty} \frac{1}{2^{k}} \max \left\{d(x(g), y(g)) ; g \in G_{k}\right\}
$$

and the maps $\sigma_{g}: Y_{\alpha} \rightarrow Y_{\alpha}, g \in G_{\infty}$ are also defined globally. In other words, if the group $G$ acts on $X$, then it acts on the spaces of pseudo-orbits as well.

In particular, if $\mathcal{F}$ is a foliation transverse to the fibres of a fibre bundle $\pi: M \rightarrow$ $B$, then its global holonomy group $H$ acts on a fibre $F$ (over a fixed point $b_{0} \in B$ ). $H$ is generated by the maps $h_{[\gamma]}$ with $[\gamma] \in \pi_{1}\left(B, b_{0}\right)$ generating the fundamental group of $B$, defined by

$$
h_{[\gamma]}(p)=\tilde{\gamma}(1),
$$

where $\tilde{\gamma}:[0,1] \rightarrow L_{p}$ is the originated at $p$ lift of $\gamma$ to the leaf $L_{p}$ through $p \in F$. 
Similarly, if $N$ is an $\alpha$-pseudoleaf and $\alpha$ is small enough, then $N$ is transverse to the fibres of our bundle and the corresponding pseudo-orbit of $H$ can be obtained by lifting to $N$ the loops generating $\pi_{1}\left(B, b_{0}\right)$.

For such a foliated bundle, a Riemannian metric on $M$ which, when restricted to $T \mathcal{F}$, coincides with the lift of a Riemannian metric on $B$, and a generating set $H_{1} \subset H$ one has the inequality

$$
\frac{1}{a} h\left(H, H_{1}\right) \leq h(\mathcal{F}) \leq \frac{1}{b} h\left(H, H_{1}\right),
$$

where $a$ (resp. $b$ ) is the maximum (resp., minimum) of the lengths of the homotopy classes $[\gamma] \in \pi_{1}\left(B, b_{0}\right)$ with $h_{[\gamma]} \in H_{1}([\mathbf{G L W}]$, p. 122). In the same way,

$$
\frac{1}{a} h_{\mathrm{ps}}\left(H, H_{1}\right) \leq h_{\mathrm{ps}}(\mathcal{F}) \leq \frac{1}{b} h_{\mathrm{ps}}\left(H, H_{1}\right)
$$

Since $h_{\mathrm{ps}}\left(H, H_{1}\right)=h\left(H, H_{1}\right)$ by Theorem 1 , inequalities (21) and (22) yield

$$
h_{\mathrm{ps}}(\mathcal{F}) \leq \frac{a}{b} h(\mathcal{F}) .
$$

Fix $m \in \mathbb{N}$ and replace $H_{1}=\left\{z_{1}, \ldots z_{s}\right\}$ by a new generating set

$$
H_{1}^{\prime}=\left\{z_{1}^{m_{1}}, z_{1}^{m_{1}+1}, \ldots, z_{s}^{m_{s}}, z_{s}^{m_{s}+1}\right\}
$$

with the exponents $m_{k}=\left[m / l_{k}\right], l_{k}$ being the length of the homotopy class $z_{k}$. For this generating set, the corresponding factor $a^{\prime} / b^{\prime}$ in (23) satisfies the inequalities

$$
\frac{m}{m+1+b} \leq \frac{a^{\prime}}{b^{\prime}} \leq \frac{m+1+b}{m}
$$

and is arbitrarily close to 1 when $m$ is sufficiently large.

This implies Theorem 2 for foliated bundles immediately.

6. An example. In $[\mathbf{B S}]$ and $[\mathbf{M i}]$, the equality

$$
h\left(G, G_{1}\right)=\lim _{\epsilon \rightarrow 0^{+}} \lim _{\alpha \rightarrow 0^{+}} \limsup _{n \rightarrow \infty} \frac{1}{n} \log N_{\alpha}(n, \epsilon)
$$

was proved for the group $G=\mathbb{Z}$ generated by $G_{1}=\{f\}$, a single transformation of $X$. Reading the proof of (24) one can observe that some of the argument is very specific: It depends strongly on the fact that the growth of $\# G_{n}, n \in \mathbb{N}$, is linear. Below, we show an example, where the equality (24) does not hold: The limit on the right hand side is strongly bigger than the entropy.

So, let $X=S^{1}, G=F_{2}$ be the free group with two generators, $f_{0}$ and $f_{1}, f_{i}$ being Morse - Smale diffeomorphisms of $S^{1}$, each of them with exactly two fixed points, a source $p_{i}$ and a sink $q_{i}$, such that $\left\{p_{0}, q_{0}\right\} \cap\left\{p_{1}, q_{1}\right\}=\emptyset$ and the sets $U_{i}=\left\{p \in S_{1}, f_{i}^{\prime}>1+\delta\right.$ on $\left.\left[p-\alpha_{0}, p+\alpha_{0}\right]\right\}, i=0,1$, cover $S^{1}$ for some $\alpha_{0}$ and $\delta>0$. Also, let $G_{1}=\left\{\mathrm{id}, f_{0}^{ \pm 1}, f_{1}^{ \pm 1}\right\}$.

Now, take any $n \in \mathbb{N}, \epsilon>0, \alpha \in\left(0, \alpha_{0}\right)$ and an $(n, \epsilon)$-separated set $A \subset S^{1}$. For any $x \in A$ and any $g=\left(g_{n}, \ldots, g_{1}\right) \in G_{n}$ with $g_{i} \in\left\{f_{0}, f_{1}\right\}$ define an $\alpha$-pseudoorbit $\tilde{x}_{g}$ of $G$ as follows:

(1) $\tilde{x}_{g}(h)=h(x)$ for all $h \in G_{n}$ and any $h \in G_{m} \backslash G_{m-1}, m>n$, of the form $h=\left(g^{\prime}, g^{\prime \prime}\right)$ with $g^{\prime} \in G_{m-n}$ and $g^{\prime \prime} \in G_{n}, g^{\prime \prime} \neq g$. 
(2) If $g(x) \in U_{0}$, then $h_{1}=f_{0}$, otherwise $h_{2}=f_{1}$, and, in both cases, $\tilde{x}_{g}\left(h_{1}, g\right)=h_{1}(g(x))+\alpha$.

(3) If $h_{1}, \ldots, h_{j} \in G_{1}$ and $\tilde{x}_{g}\left(h_{j}, \ldots, h_{1}, g\right)$ are already defined, and $\tilde{x}_{g}\left(h_{j}, \ldots, h_{1}, g\right) \in U_{0}$, then $h_{j+1}=f_{0}$, otherwise $h_{j+1}=f_{1}$, and, in both cases,

$$
\tilde{x}_{g}\left(h_{j+1}, h_{j}, \ldots, h_{1}, g\right)=h_{j+1}\left(\tilde{x}_{g}\left(h_{j}, \ldots, h_{1}, g\right)\right)+\alpha .
$$

(4) If $j \in \mathbb{N}, m \in \mathbb{N}, h=\left(h_{m}^{\prime}, \ldots, h_{1}^{\prime}, h_{j}, \ldots, h_{1}, g\right)$ with $h_{i}^{\prime} \in G_{1}, h_{1}^{\prime} \neq h_{j+1}$ and $h_{1}^{\prime} \neq h_{j}^{-1}$, then $\tilde{x}_{g}(h)=h_{m}^{\prime} \circ \cdots \circ h_{1}^{\prime}\left(\tilde{x}_{g}\left(h_{j}, \ldots, h_{1}, g\right)\right)$.

Roughly speaking, $\tilde{x}_{g}$ is obtained by a suitable modification of the orbit $G(x)$ along a single originated at $g$ branch of the Cayley graph of $G$.

A standard induction involving Mean Value Theorem shows that

$$
d\left(\tilde{x}_{g}\left(h_{j}, \ldots h_{1}, g\right), h_{j} \circ \cdots \circ h_{1} \circ g(x)\right) \geq \alpha \cdot(1+\delta)^{j} \geq \epsilon
$$

whenever $j>n(\epsilon, \alpha), n(\epsilon, \alpha)$ being the largest integer which does not exceed the quotient

$$
\log \frac{\epsilon}{\alpha} / \log (1+\delta) .
$$

It follows that the set $A_{\alpha}$ of all such pseudo-orbits $x_{g}$ is $(n+n(\epsilon, \alpha)+1, \epsilon)$-separated. In fact, if $x \neq x^{\prime} \in A$, then $d\left(\tilde{x}_{g}(h), \tilde{x}_{g^{\prime}}^{\prime}(h)\right)=d\left(h(x), h\left(x^{\prime}\right)\right) \geq \epsilon$ for some $h \in G_{n}$; if $x=x^{\prime}$ but $g \neq g^{\prime}$, then $\tilde{x}_{g^{\prime}}\left(h_{j}, \ldots, h_{1}, g\right)=h_{j} \circ \cdots \circ h_{1} \circ g(x)$ and the inequality $d\left(\tilde{x}_{g^{\prime}}(h), \tilde{x}_{g}(h)\right) \geq \epsilon$ for some $h \in G_{n+n(\epsilon, \alpha)+1}$ follows from (25). Clearly,

$$
\# A_{\alpha}=2^{n} \cdot \# A \text {. }
$$

This yields the inquality

$$
N_{\alpha}(n+n(\epsilon, \alpha)+1, \epsilon) \geq 2^{n} \cdot N\left(n, \epsilon, S^{1}\right)
$$

for all $n, \epsilon>0$ and $\alpha<\alpha_{0}$. Passing to suitable limits we end up with

$$
\lim _{\epsilon \rightarrow 0^{+}} \lim _{\alpha \rightarrow 0^{+}} \limsup _{n \rightarrow \infty} \frac{1}{n} \log N_{\alpha}(n, \epsilon) \geq h\left(G, G_{1}\right)+\log 2 .
$$

Inequality (26) shows that, what was already observed in [GLW], the entropy of a group (a pseudogroup, a foliation) depends strongly on the growth of the group (the orbits, the leaves). It would be very interesting to obtain a good upper estimate of the right hand side in $(24)$ in terms of $h\left(G, G_{1}\right)$ and the growth of $G$. Some indication in this direction is contained in the proof of Proposition 6.13 in [GLW].

\section{REFERENCES}

[BS] M. Barge and R. Swanson, Pseudo-orbits and topological entropy, Proc. Amer. Math. Soc. 109 (1990), 559-566.

[BK] P. Buser and H. Karcher, Gromov's almost flat manifolds, Astérisque 81 (1981), 1-148.

[Eg] S. Egashira, Expansion growth of foliations, Ann. Fac. Sci. Univ. Toulouse 2 (1993), $15-52$. 
[GLW] E. Ghys, R. Langevin and P. Walczak, Entropie géométrique des feuilletages, Acta Math. 160 (1988), 105-142.

[HH] G. Hector and u. Hirsch, Introduction to the Geometry of Foliations, Part $A$ and B, Vieweg and Sohn, Braunschweig, 1986 and 1987.

[Hu1] S. Hurder, Ergodic theory of foliations and a theorem of Sacksteder, Dynamical Systems: Proceedings, University of Maryland 1986 - 87. Lecture Notes in Math., vol 1342, Springer Verlag, Berlin and New York, 1988, pp. 291-328.

[Hu2] S. Hurder, Exceptional minimal sets of $C^{1+\alpha}$ group actions on the circle, Ergodic Th. \& Dynam. Sys. 11 (1991), 455-467.

[Hu3] S. Hurder, Coarse geometry of foliations, Geometric Study of Foliations, Proceedings, Tokyo 1993, World Sci., Singapore, pp. 35-96.

[Hur] M. Hurley, On topological entropy of maps, Ergodic Th. \& Dynam. Sys. 15 (1995), $557-568$.

[In] T. Inaba, Expansivity, pseudoleaf tracing property and topological stability of foliations, Preprint 1995.

[IT] T. Inaba and N. Tsuchiya, Expansive foliations, Hokkaido Math. J. 21 (1992), 39-49.

[LW1] R. Langevin and P. Walczak, Entropie d'une dynamique, C. R. Acad. Sci. Paris, Sér. I 312 (1991), 141-144.

[LW2] R. Langevin and P. Walczak, Entropy, transverse entropy and partitions of unity, Ergodic Th. \& Dynam. Sys. 14 (1994), 551- 563.

[Mi] M. Misiurewicz, Remark on the definition of topological entropy, Dynamical Systems and Partial Differential Equations (Caracas 1984), Caracas, 1986, pp. 65-67.

Instytut Matematyki, Uniwersytet Łódzki, Banacha 22, 90238 Łódź, Poland

E-mail address: andbis@ krysia.uni.lodz.pl and pawelwal@ krysia.uni.lodz.pl 